\title{
La verdad de la Naturaleza ${ }^{1}$
}

\author{
EDGAR MARAGUAT \\ Universitat de València
}

Esta contribución está dedicada a la comprensión de la relación que asume Hegel entre la Naturaleza y el Espíritu (una cuestión que es para la Antropología filosófica preliminar). Naturaleza y Espíritu son los temas de las dos ciencias filosóficas reales del sistema hegeliano (la Philosophie der Natur y la Philosophie des Geistes), que se añaden, completándolo, a la Lógica o ciencia pura.

Hegel escribe al comienzo de la parte dedicada en su Sistema a la Filosofía del Espíritu que la Naturaleza es la presuposición del Espíritu y el Espíritu la verdad de la Naturaleza:

"Para nosotros el espíritu tiene a la naturaleza como presuposición suya, de la cual él es la verdad y, por tanto, lo absolutamente primero de ella. En esta verdad ha desaparecido la naturaleza ..." (G. W. F. Hegel 1830, § 381; cf. §§ 222, 251, $376,388)$.

¿Qué significa, sobre todo, que el Espíritu es la verdad de la Naturaleza? ¿Y qué que la Naturaleza ha "desaparecido" o es sólo una presuposición del Espíritu? Quisiera tentar a cierta respuesta a estas cuestiones por medio de tres aproximaciones de carácter histórico-filosófico ${ }^{2}$.

1 * La investigación que ha propiciado este trabajo ha sido auspiciada por el Proyecto de Investigación "Filosofía del Lenguaje, de la Lógica y de la Cognición" que financia el (otrora) Ministerio de Educación y Ciencia (Consolider-C HUM2006-08236).

2 En lo que sigue no voy a atender a las distinciones hegelianas, no difíciles de reconstruir (al menos a grandes rasgos), entre Inteligencia, Espíritu y Alma (tampoco es éste un estudio de la evolución de las nociones en su obra). Espíritu se toma aquí en el sentido de la Philosophie des Geistes de la obra madura, que abarca el alma, la conciencia, la razón, la inteligencia, el espíritu libre, el espíritu de un pueblo, el espíritu del tiempo y tantas otras cosas. Asumo que esta decisión no frustra el propósito de estas páginas. 
En primer lugar, quisiera recordar que Hegel debuta en la escena filosófica con su escrito sobre la Diferencia entre los sistemas de Fichte y Schelling (1801) proponiendo una suerte de rehabilitación de Spinoza y abogando concretamente por que el Sistema de la Filosofía sea un Sistema de lo Absoluto con dos fenómenos de igual rango o dignidad y realmente diferentes (o realmente opuestos), a saber, la Inteligencia y la Naturaleza (es, por cierto, el propio Hegel quien asemeja la Inteligencia y la Naturaleza a los atributos del pensamiento y la extensión, atributos de la sustancia única en el sistema de Spinoza; cf. G. W. F. Hegel 1801, p. $71^{3}$ ).

Hegel expresa este ser fenómenos realmente diferentes u opuestos de lo Absoluto diciendo que lo Absoluto se da por entero en ambos (de modo que no son sus componentes) y que ambos opuestos están cancelados al estar sentados como idénticos (cf. G. W. F. Hegel 1801, p. 63) o al estar en ambos lo Absoluto (p. 66). Aunque Hegel no dice nada muy explícito en 1801 sobre la relación entre Inteligencia y Naturaleza, que ambos sean fenómenos realmente diferentes de lo Absoluto parece excluir tanto la causalidad en ambos sentidos (como para Spinoza, ni la naturaleza sería un efecto del espíritu ni el espíritu un efecto de la naturaleza) como que la relación sea la relación entre una sustancia y sus accidentes (ni la naturaleza sería sustancia y el espíritu su accidente, ni viceversa).

Explícitamente el escrito sobre la Diferencia excluye al menos el dualismo, las "unilateralidades" complementarias del idealismo y el materialismo, y la mezcla o mezcolanza (Vermengen) de Inteligencia y Naturaleza que en la época representaba la "teoría de las fibras" de la conciencia de Charles Bonnet (cf. ibid., p. 68). El punto de vista "superior" (que Hegel asume), que viene a ser la única (verdadera) solución que resta, sería el que reconoce en las ciencias de la Naturaleza y la Inteligencia, por igual, "el mismo Absoluto". Dentro de cada una de ellas, según la exposición de 1801, su tema propio o principio (la Inteligencia en el caso de la Filosofía Trascendental, la Naturaleza en el caso de la Naturphilosophie) se comporta como lo sustancial, mientras su opuesto aparece como lo accidental. Ahora bien, sobre cómo se comportan recíprocamente fuera de esas ciencias o, dicho de otro modo, desde el punto de vista de lo Absoluto mismo, el escrito calla.

En sentido afirmativo, en este "Sistema de la Identidad" de la primera hora

3 La inspiración de Spinoza es, por cierto, decisiva en la constitución del llamado Idealismo Alemán en general: Fichte y el primer Schelling proponen continuar la revolución kantiana promulgando un spinozismo de la subjetividad y como un racionalismo spinozista (ya no "de la subjetividad") debe tomarse la Exposición de mi sistema de Schelling, de 1801, que el escrito sobre la Diferencia en cierto modo anticipa. 
la relación que investigamos parece haber colapsado en lo que hoy llamaríamos una "Teoría de la Identidad" (lo que en cierto sentido es Naturaleza, en otro sentido es Espíritu). De ser así, el desarrollo del sistema maduro (la doctrina, concretamente, de que el Espíritu es la verdad de la Naturaleza) habría que verlo corrigiendo o al menos matizando esa identidad. En efecto, las ciencias de la Naturaleza y el Espíritu en el sistema maduro no parecen ya ser simplemente complementarias, ni en sentido estricto ciencias de igual rango (todo ello tiene que ver con la autosuficiencia ganada por la Lógica y el carácter circular de la Enciclopedia, pero aquí no puedo discutir esta cuestión arquitectónica).

No obstante, en el sistema maduro Naturaleza y Espíritu siguen siendo modos (o "elementos") en que se expone el mismo Absoluto (lo que entonces es denominado la "Idea"), así que sigue abierta la cuestión de cómo se relacionan esos modos en que se da lo mismo (cf. G. W. F. Hegel 1830, § 18).

\section{III}

En segundo lugar quisiera llamar la atención sobre el modo aristotélico en que en la Enciclopedia (en el lugar al que aludí al comienzo) se presenta la relación.

Hegel indica allí, como vimos, que, aunque el Espíritu presupone la Naturaleza, el Espíritu es absolutamente primero con respecto a la Naturaleza. Signifique esto lo que signifique, hemos de suponer que esta relación no puede conllevar una recaída en alguna de las posiciones que el spinozismo de la primera hora había evitado, en particular en un idealismo de algún tipo ${ }^{4}$.

En mi opinión, el parágrafo enciclopédico evoca la relación aristotélica de tipo orgánico entre los elementos de un todo y el todo mismo, como por ejemplo la relación entre la mano y el cuerpo, pero también la relación (política y orgánica) entre el ciudadano y la ciudad. Aristóteles sentencia en el célebre comienzo de su Política que la mano, que, por supuesto, compone el cuerpo o es un elemento del cuerpo (y en ese sentido el cuerpo presupone la mano), sólo es mano conectada al cuerpo (y que, análogamente, el ciudadano es ciudadano en virtud de su pertenencia a la ciudad, si bien la ciudad existe en y por sus ciudadanos).

Aunque la expresión no sea aristotélica, podríamos decir que mano y ciudadano tienen su verdad en el cuerpo y la ciudad, respectivamente, pues eso que es la mano o el ciudadano sólo lo es en el cuerpo o la ciudad, respectivamente. El

4 Para Pippin, al contrario que para mí, en la letra de este párrafo no resuenan otros planteamientos y soluciones del problema: "Este pasaje carece de resonancias contemporáneas e incluso históricas, pues Hegel está claramente inventando los términos en los que plantear el problema y sugerir su solución" (1999, p. 197). No obstante, como otros intérpretes, percibe en lo que él considera la solución hegeliana la sintonía de fondo con Aristóteles, que, a mí, por las razones que ahora expondré, me parece notoria. 
sentido aristotélico del texto de Hegel sugiere, pues, que Naturaleza y Espíritu se relacionan como las partes y el todo en un todo orgánico.

Hegel, por tanto, no trata al Espíritu y la Naturaleza en el desarrollo del Sistema ni como cosas capaces de interactuar causalmente (a la manera de cierto dualismo incoherente), ni como siendo uno el fenómeno o manifestación del otro, ni como siendo Naturaleza y Espíritu, simplemente, aspectos diversos de lo mismo (como para la Teoría de la Identidad), sino, más bien, como siendo la Naturaleza el elemento del Espíritu y el Espíritu la totalidad en la que lo natural, por así decir, toma forma y determinación. Ahora bien, cómo ocurre esto último, o en qué sentido el Espíritu es esa totalidad para la Naturaleza, está por ver.

\section{IV}

En tercer lugar quisiera primero recordar una generalidad de los tránsitos en el sistema hegeliano. Cuando en su obra lo que sigue aparece como verdad de lo anterior, ello no ocurre nunca en el sentido de que lo anterior hubiera sido injustamente asumido y ahora fuera desplazado o, mejor, reemplazado, sino en el sentido de que lo posterior muestra cómo debía ser tomado o entendido aquello ${ }^{5}$.En ese sentido, la secuencia Naturaleza-Espíritu es una secuencia que ha de llevar a comprender la Naturaleza. Ahora bien, ¿de qué modo?

Hegel asume, desde luego, siguiendo en esto a Schelling, el pensamiento de que la Naturaleza es algo vivo, atravesado en sí mismo por fuerzas y finalidades, pensamiento que él opone a la representación kantiana y fichteana de una naturaleza (que podemos escribir con minúsculas) que es pura exterioridad o extensión, pura indeterminación, en la que todo concepto o finalidad es producido o proyectado por los observadores (por su facultad de juzgar).

He de recordar en este punto, brevemente, cómo recibe Hegel la doctrina kantiana de lo vivo y los fines naturales. Como es sabido, Hegel echa en cara a Kant haber rebajado la idea (bien captada y rescatada de Aristóteles) de lo vivo como un fin en sí mismo, idea que los tres comparten, a un mero compromiso práctico-heurístico (cf. G. W. F. Hegel 1830, § 58, entre otros muchos lugares).

A mi modo de ver, la posición de Hegel es, por el contrario, la de que lo que es un mecanismo desde cierto punto de vista es por sí mismo un órgano que sirve a una función, pues son las relaciones mecánicas las que están sirviendo a un fin a mayor escala, según se puede apreciar desde una perspectiva

5 Un buen ejemplo de este carácter de los tránsitos lo ofrece el paso en la Fenomenología del Espíritu de la Conciencia a la Autoconciencia ("no sólo la conciencia de las cosas no es posible sino para una autoconciencia, sino que precisamente ésta y solamente ésta es la verdad de esas figuras [de la Conciencia: sensación, percepción, entendimiento; E. M.]”, G. W. F. Hegel 1807, p. 271, mi énfasis). 
ventajosa. De ahí que podamos decir que la perspectiva de los fines sobre la Naturaleza revela aquello que la Naturaleza es capaz de hacer de suyo y, por tanto, es en sí misma ${ }^{6}$.

Puesto que, por otro lado, Hegel abraza la idea kantiana de que el Espíritu es fin en sí mismo por antonomasia, podemos interpretar entonces el modo en que el Espíritu es la verdad de la Naturaleza explicando que comprendemos que la Naturaleza está viva y llena de fines, porque, previamente, por familiaridad con nosotros mismos, sabemos qué es tener uno mismo un fin. $\mathrm{O}$, dicho de otro modo, puesto que los hombres son seres, como dice Kant, que obran según la representación que tienen de cómo deben ser las cosas (de un estado de cosas a alcanzar), están en condiciones de apreciar que la naturaleza está sujeta a normas o realiza un concepto, aunque ella misma no lo sepa.

Quisiera insistir en este punto decisivo. Contra lo que suele creerse, Hegel suscribe la tesis kantiana de que la finalidad en la Naturaleza queda sólo a la vista de un observador que es un fin para sí mismo. En efecto, Hegel afirma expresamente en la Fenomenología, en el lugar en que esto se discute por primera vez, que la vida (en la Naturaleza) es vida sólo para esa otra vida que llamamos Autoconciencia (el elemento del Espíritu) ${ }^{7}$. Pero Hegel, al contrario que Kant, entiende que este quedar a la vista o ser puesto en claro no implica que los fines que nos parecen obvios en los organismos son puramente fines aparentes.

La plausibilidad de su posición depende de que mecanismo y teleología dejen de verse como modos rivales de funcionar objetivamente que segregarían unos objetos de otros (los objetos mecánicos de los objetos teleológicos, por así decir). Y la única manera de anular esa rivalidad es elevarse a la concepción de que, como dije antes, lo que desde cierto punto de vista se comporta mecánicamente desde otro punto de vista se conduce teleológicamente (a admitir, por ejemplo, que cierta descarga eléctrica y contracción muscular, repetidas rítmicamente, por los efectos que tienen, desempeñan la función de un bombeo).

6 No puedo discutir aquí, como es debido, esta interpretación. Quiero sólo reconocer que soy consciente de que éste no es el sentido que parece tener siempre lo que Hegel llamaría "la verdad del mecanismo" (cf. G. W. F. Hegel 1816, pp. 437 s. y 440).

7 El paso quizá más elocuente forma parte de la introducción al Capítulo iv de la obra: "Esa segunda unidad es el simple género [Gattung], el cual, en el movimiento de la vida misma, no existe para sí mismo como tal cosa simple; sino que en ese resultado la vida remite a algo otro que lo que ella misma es, a saber: a la conciencia para la que la vida es como dicha unidad, o para la que la vida es como género" (G. W. F. Hegel 1807, p. 283). El género, que es el fin último del organismo, no existe para sí mismo como algo simple; es algo simple o existe como tal sólo para la conciencia. Más adelante, en el capítulo sobre la "Razón observadora", Hegel compara de nuevo los "fines reales" (los organismos que se conservan por sí mismos) con la (auto)conciencia: "en la observación de la naturaleza orgánica, la autoconciencia no encuentra otra cosa que esa esencia, se encuentra a sí como cosa, como una vida" (p. 367). 
Puede decirse, es cierto, que hay definitivamente en esta solución un grano de Filosofía de la Identidad.

De lo anterior podemos concluir que el Espíritu es la verdad de la Naturaleza no porque lo que parecía Naturaleza era Espíritu o porque el Espíritu ocupa el lugar, de algún modo, de la Naturaleza, sino porque la aparición del Espíritu deja ver la Naturaleza a una nueva luz (deja ver lo mecánico como teleológico) ${ }^{8}$.

\section{V}

Éste puede ser el sentido en el que, bajo la inspiración de Aristóteles y Kant, Hegel asume que el Espíritu es la Totalidad en la que la Naturaleza reluce bajo su verdadera determinación. Que el Espíritu sea, como dice Hegel, "el alma del mundo", "la sangre universal ... presente en todas partes" (cf. G. W. F. Hegel 1807 , p. 266) significaría que hay fines por doquier, también en la Naturaleza, siendo que llamamos Espíritu al ser en que la realización de los fines es una realización que se hace explícita.

Concluyo. Creo que estas tres aproximaciones conducen a interpretar la relación entre Naturaleza y Espíritu en el sistema hegeliano maduro como una relación de identidad y diferencia, concebida fundamentalmente bajo la inspiración aristotélica de la noción de totalidad orgánica en que los elementos toman su determinación del todo dinámico que constituyen. La naturaleza que ha desaparecido, en este cuadro, es la naturaleza muerta del mecanicismo, a la que se reduce la naturaleza de la filosofía trascendental. La naturaleza que no cumple un fin, o lo hace precariamente, cede su lugar en el Sistema de Hegel a una Naturaleza que es el elemento en que se realizan los fines y, con ello, el elemento en que se realiza el Espíritu, pero no como una materia que es potencialmente cualquier cosa, sino como una materia que puede ser vista como conteniendo en sí misma esos fines.

8 En realidad, como ha subrayado Wolff, ésta no es toda la verdad de la recepción crítica de la doctrina kantiana de la teleología. Hegel piensa que Kant se queda radicalmente corto al convertir la finalidad interna en una máxima de la comprensión de la naturaleza, pero que, por otro lado, es demasiado optimista al pensar que los seres vivos aparecen simplemente como fines de sí mismos. Hegel considera, más bien, que el estudio de lo que él llama el "proceso del género" revela la "impotencia de la naturaleza", esto es, la precariedad de la realización de los fines en la naturaleza, donde los seres están entregados a diversas fuerzas que los descoyuntan y terminan por darles muerte (cf. M. Wolff 1992, p. 134). Este importante matiz puede conducirnos a interpretar que el Espíritu es la verdad de la Naturaleza en el sentido de que en él se consuma lo que en la Naturaleza no llega a cumplirse. Pero sería un disparate que esta interpretación nos devolviera al dualismo del que estábamos huyendo. 


\section{REFERENCIAS}

ARISTÓTELES, 1989: Política. Madrid: Centro de Estudios Constitucionales (trad. de Julián Marías y María Araújo).

HEGEL, G. W. F., 1801: Diferencia entre el sistema de filosofía de Fichte y el de Schelling. Madrid: Alianza, 1989 (trad. de Juan A. Rodríguez Tous). 1807: Fenomenología del Espíritu. Valencia: Pre-Textos, 2007 (trad. de Manuel Jiménez Redondo).

1816: Wissenschaft der Logik, II, en: Werke, 6. Frankfurt am Main: Suhrkamp, 1969.

1830: Enciclopedia de las ciencias filosóficas en compendio. Madrid: Alianza, 1997 (trad. de Ramón Valls Plana).

PIPPIN, R. B., 1999: "Naturalness and Mindedness: Hegel's Compatibilism", European Journal of Philosophy, 7 (2), pp. 194-212.

WOLFF, M., 1992: Das Körper-Seele-Problem. Kommentar zu Hegel, Enzyklopädie (1830), § 389. Frankfurt am Main: Vittorio Klostermann. 
